\title{
Implicit responses in discrimination learning of retardates'
}

\author{
Harold J. Fleteher \\ UNIVERSITY OF WISCONSIN
}

\begin{abstract}
Abstraet
15 severely retarded Ss received 12 sessions of 4 12-trial object discrimination problems differentiated by the following procedures enforced during the first 6 trials only: a prompt (light cue in front of correct stimulus) appeared or did not, and an instrumental response was permitted or was not. Subsequent intraproblem performance indicated (1) significant learning when only the prompt appeared and no response was permitted, and (2) more learning with prompt plus response than with response alone (conventional trialand-error procedure). MA was correlated significantly with performance on prompt-only problems but not with trial-and-error performance. These data suggested a differentiation between implicit and explicit response learning processes.
\end{abstract}

\section{Problem}

In a previous report (Fletcher et al, 1965), monkeys, retardates, and preschool children were tested on two 36-trial object discrimination problems, one involving prompting during the first six trials only, and the other involving conventional trial-and-error procedures during all trials. Data from trials 7-36 indicated that human Ss learned significantly more during prompting than they did during $\mathrm{e}$ i $\mathrm{t}$ he $\mathrm{r}$ the same number of conventional discrimination trials or during as many conventional trials as were necessary to make six correct responses. Because the number of rewarded overt responses could not account for the superiority of prompting, it was suggested that the prompt elicited an implicit response sequence which involved the prompted object, was independent of the explicit instrumental response which followed, and functioned as did an ordinary trial. Specifically, it was suggested that the sheer occurrence of the implicit response increased the tendency to respond overtly to the prompted object, and that the strength of this tendency was independent of the same tendency which increased as a function of rewarded instrumental responses.

Ordinarily both responses occur on a prompted trial, and it is manifestly impossible to assess the effects of either alone. The present experiment was designed to permit such an evaluation, however, by providing trials during which only an implicit response could occur. Any observed learning would then be attributed to implicit responses alone.

\section{Method}

Nineteen severely retarded males (with previously mentioned experience) were tested in a modified WGTA in which $\mathrm{E}$ sat behind a one-way mirror, raised a door below the mirror, and pushed a presentation tray toward S. The tray, made completely from opaque white acrylic plastic, contained two channels in which slid $4 \times 4$ in white plastic bases. In their forward position the bases butted against the front edge and covered two foodwells 10 in apart. In the tray's front edge, $21 / 2$ in high and angled $45^{\circ}$ from horizontal, were two 1 in jewelled amber lights (prompts) centered directly in front of the foodwells. Multidimensional junk objects were attached to the bases, and $S$ was required to push the object back to expose a foodwell.

Although familiar with the apparatus and procedures, Ss were first required to demonstrate the appropriate response to the prompt. Two identical grayblocks were presented in four 12-trial blocks during which the prompt (light) was lighted in front of the rewarded foodwell, and $S$ was required to make 11 correct responses in any of the 12-trial blocks. For these and subsequent sessions, rewarded position was randomized but balanced over blocks of six trials, raisin rewards were used, the noncorrection procedure was employed, and verbal instructions were limited to essentially "find the raisin."

The 12-session test phase consisted of four different 12-trial object discrimination problems per daily session. The problems were defined by the following procedures enforced during the first six trials only:

PR Problems: prompt indicating correct object; instrumental response permitted in the presence of the prompt.

R Problems: no prompt; instrumental response permitted (conventional trial-anderror procedure).

P Problems: prompt on; no response permitted; Ss told on each trial "look at but don't touch the objects"; (Objects were actually locked, but all Ss followed instructions).

C Problems: control; no prompt; no response; Ss given same instructions as in $\mathrm{P}$.

The order of the four daily problems was randomized for each $\mathrm{S}$ independently. For all problems Trials 7-12 followed without interruption but with conventional trialand-error procedures only.

\section{Results}

A preliminary examination of overall performance on Trials 7-12 for all problems revealed that four Ss failed to evidence an arbitrary minimal level of learning (60\% correct), and these data were rejected. Subsequent analyses were therefore made on the data 
Table 1. Percent of correct responses over all sessions

\begin{tabular}{lccccc} 
& \multicolumn{4}{c}{ Type of } & Problem \\
& PR & R & P & C \\
& & & & \\
Trials $1-6$ & 91 & 74 & - & - \\
Trial 7 on1y & 87 & 83 & 58 & 50 \\
Trials 7-12 & 87 & 87 & 77 & 73
\end{tabular}

from 15 Ss (MA's 26-67 mos.; median 48.5). ${ }^{2}$ The essential results are presented in Table1.

The control exerted by the prompt is evident in the Trials 1-6 data; PR performance was $91 \%$ while conventional unassisted trial-and-error performance ( $R$ problems) was only $74 \%$ over the same trials. The favorable comparison of this latter performance to the $73 \%$ under the control condition (Trials 7-12) contraindicated any artifactual effects of merely not permitting an instrumental response for six trials.

Trial 7 data represented the purest measure of learning which took place under each experimental condition. Because of the nature of the hypotheses investigated, only selected, one-tailed tests were made. Consistent with previous results, these data indicated that more learning took place during prompted trials than during conventional discrimination trials (PR vs. $\mathrm{R})$, but the $4 \%$ difference was significant at only the .10 level $(t=1.367 ; d f=14)$. The critical comparison, $P$ vs. $C$, revealed unequivocally that prompting alone was sufficient for learning to occur $(t=2.354$; $d f=14$; $\mathrm{p}<$.025).

MA scores were correlated with overall correct Trial 7 responses on both $R$ and $P$ problems. As is frequently the case, a low and insignificant (Spearman Rank) correlation coefficient of .175 was obtained when MA was related to performance on $\mathrm{R}$ problems, performance which reflects the amount learned under trial-and-error procedures used in most learning research. The correlation with performance on $\mathbf{P}$ problems, however, was .535 $(\mathrm{p}<.05)$.

Trials 7-12 data provided only indirect measures of the effects because of the obvious confounding with trial-and-error learning. The initial $4 \%$ differential was overcome as performance on $\mathrm{R}$ problems reached the apparent $87 \%$ asymptote evidenced under PR problems. The critical $P$ vs. C comparison revealed again a small but significant $4 \%$ advantage following prompting $(t=1.924 ; \mathrm{df}=14 ; \mathrm{p}<.05) ;$ actually only three of the 15 scores were higher under the $C$ problems $(p=.018)$.

\section{Results}

These results support the interpretation that the prompt elicited reliably and accurately an implicit response sequence which involved the prompted object and functioned as as an independent trial, i. e., six implicit responses alone were sufficient for learning to occur. When both implicit and explicit responses were allowed, there remained a small amount of learning not accounted for by overt response learning.

These data extend other research concerned either directly or indirectly with eliciting implicit (attentional, orienting, etc.) responses to the correct stimulus. The effects of various procedures on nonhuman primate learning have been demonstrated, for example, by Harlow (1944) and Fletcher \& Takemura (1965). With retardates of virtually identical MA's, House (1964) has recently explored a Trial 1 "demonstration" procedure of holding up the object and the reward (or exposing the empty foodwell) and verbalizing the reward contigency. While lower than that following conventional Trial 1 procedures, Trial 2 performance following demonstration was considerably above chance and clearly indicated that overt response and reward were not necessary for learning to occur. The present study confirms implicit response learning and demonstrates further that appropriate prompting procedures obviate both manipulation of the correct object and presentation of the reward.

Finally the correlational data suggest that the processes measured by intelligence tests are related, if not identical, to those which are involved in implicit response learning but not to those which are utilized in conventional trial-and-error learning.

\section{References}

FLETCHFR, H. J., DAVIS, J. K., ORR, B. M., \& ROSS, P. Prompted versus trial-and-error initial object discrimination learning by monkeys, retardates, and preschool children. Psychon. Sci., 1965, 2, 59-60.

FLETCHER, H. J., \& TAKEMURA, K. Prompted versus trial-anderror concept discrimination learning in monkeys. Percept. mot. Skills, 1965, in press.

HARLOW, H. F. Studies in discrimination learning by monkeys: II. Discrimination learning without primary reinforcement. J. gen. Psychol., 1944, 30, 13-21.

HOUSE, B. J. Discrimination learning without overt response or reward in retardates. Amer. J. ment. Defic., 1964, 68, 734-740.

\section{Notes}

1. The author wishes to thank Harvey A. Stevens and Ronald Sindberg of the Central Wisconsin Colony administration for their cooperation and particularly Mrs. Mary McNamee and Mr. Frank Vojtik for their assistance.

2. MA scores were obtained within a two-week period by Mrs. Judy Yaeger using the Peabody Picture Vocabulary Test, Form A. 